\title{
The Effect of Oral Care and Chlorhexidine on the Rate of Ventilator Associated Pneumonia (VAP)
}

\author{
Mohammed Fathudeen Zakri ${ }^{1}$, Salah Hussain Shammakhi ${ }^{1}$, Ghadeer Hassan Ajlan ${ }^{1}$, \\ Majed Yahia Sabei ${ }^{1} \&$ Mohammed Abdulrahman Zurbotan ${ }^{1}$ \\ ${ }^{1}$ Ministry of health (MOH), Sabya General Hospital, Nursing Department, Sabya, Saudi Arabia \\ Correspondence: Mohammed Fathudeen Zakri, Ministry of health (MOH), Sabya General Hospital, Nursing \\ Department, Sabya, Saudi Arabia.
}

Received: November 21, 2019 Accepted: December 22, 2021 Online Published: December 30, 2021

doi:10.5539/gjhs.v14n2p30

URL: https://doi.org/10.5539/gjhs.v14n2p30

\begin{abstract}
Objective: Ventilator-associated pneumonia is considered major pneumonia, which develops in the intensive care unit patients following mechanical ventilation for about two days. This study reviews oral care and chlorhexidine effect on ventilator associated pneumonia occurrence.

Methods: A critical review approach was adopted where publications from 2007 to 2017 were considered. These publications were gathered from electronic searches through the different databases, for instance, MEDLINE, EMBASE, CINAHL, and Cochrane Library. This protocol was used for the selection of the studies and their analysis.

Results: The review showed that $0.12 \%$ Chlorhexidine use assists in reducing bacterial growth. It also suggests that the use of affirmative outcomes for mechanically ventilated patients, including improved patient outcomes, decreased in the duration of the patients' hospital stays, and reduced health care cost.
\end{abstract}

Keywords: chlorhexidine, oral care, rate of Ventilator Associated Pneumonia

\section{Introduction}

Studies have shown that ventilator-associated pneumonia (VAP) accounts for nine to forty percent of the infections in ICU (Intensive Care Unit) (Torres et al., 2017; Wei \& Yang, 2019). De Lacerda Vidal et al. (2017) stated that VAP occurrence is related to the increased hospital stay, enlarged hospital expenditure as well as higher mortality and morbidity rate. International Nosocomial Infection Control Consortium (INICC) epidemiological study reported that VAP incidence accounts for medical surgical ICUs is 13.1 in 100 mechanical ventilator-days, while it is 9.02 for NICUs (Rosenthal et al., 2016). Empirical evidence highlights massive VAP costs in the US and Europe, costing about \$20,000 (Asehnoune et al., 2014; Wei \& Yang, 2019). This shows the significance of clinical analysis of VAP incidence and is viewed as a strategy for improving ICU care.

Most studies highlight VAP as a substantial risk factor, which is linked to curarization and sedation, heart, regurgitation, aspiration as well as the lung-associated disease (Sousa, Ferrito, \& Paiva, 2018; Torres et al., 2017; Wei \& Yang, 2019). ICU patients also report pathological changes in the oral cavity, which include periodontal disease relief, relief from periodontal disease, oral mucosal lesions, dryness of lips and mucous membranes, fungal infections, increased biofilm on the oral surface, and more (Wei \& Yang, 2019). Also, critically ill patients rely on others to ensure their oral hygiene. The oral health of these patients substantially deteriorates in case adequate care is not provided. The outcome of ineffective care includes the dental plaque colonization of microbial pathogens, which might be causative of respiratory infections (Hua et al., 2016).

For the majority of ICU treated patients, mechanical ventilation is necessary as they are unable to breathe on their own (Mauk, 2010). This life-preserving technique requires a pump outside the body, which provides oxygen to the lungs. This process is open to microbial contamination, which can cause infections and prolong hospital stays. The risk for the VAP is higher for ventilator patients which results in the transfer of bacteria to the respiratory tract through the endotracheal tube (Munro \& Grap, 2011). As a part of the oral cavity's normal flora, microorganism, and dental plaque develops a perfect environment for the growth of microbial. Wunsch et al. (2010) reported that individuals over 65 years of age comprise 52.2 percent of the ventilated patients as well as co-morbid conditions which reduces the survival rate. 
Ventilator-associated pneumonia is one possible complication for ventilated patients. For reducing the VAP occurrence, and thus raise patient survival rates, a VAP bundle can be employed, consisting of chlorhexidine use; toothbrushing; raising beds to a 30-degree angle, or greater; replacing nasogastric tubes with oral gastric tubes, and sedation vacations (regular periods of reduced sedation). A critical literature review is provided regarding oral care efficacy and chlorhexidine gluconate (CHX) as a method of reducing VAP in ventilated treated patients.

\section{Methods}

\subsection{Study Design and Sample}

A qualitative study design is used, where a critical review approach is applied. Accordingly, the articles for review were selected, which ranged from 2007 to 2017. These articles were selected from reliable sources, including MEDLINE, EMBASE, CINAHL, and Cochrane Library. The researches also accessed the relevant guidelines, policies as well as governmental publications along with national and international published statistics. World Health Organization reports were also consulted.

\subsection{Studies Selection}

The selection of the articles was based on the determined inclusion and exclusion criteria (Table 1). Only primary sources in English that involved adult ICU patients and were published between 2007 and 2017 were included in this review. Papers meeting these criteria could be considered, regardless of the experimental design implemented therein.

Table 1. Study Inclusion and Exclusion Criteria

\begin{tabular}{lll}
\hline Criteria & Inclusion Criteria & Exclusion Criteria \\
\hline \multirow{3}{*}{ Design } & Randomized Controlled Trials (RCTs) & Case Report \\
& Clinical Trials & Blogs \\
& Prospective Studies & Case Series Studies \\
& Retrospective Cohort Studies & \\
\hline Time & Ranging from 2007 to 2017 & Older than 2007 \\
\hline Language & English & Articles other than English \\
\hline Type of Intervention & Have been conducted on individuals & Have been conducted on animals \\
\hline Follow-up Time & At least two years & $>$ two years \\
\hline
\end{tabular}

\subsection{Study Search}

A literature review was conducted utilizing several databases, including MEDLINE, EMBASE, CINAHL, and Cochrane Library. The researcher also used various keywords for deriving the relevant articles from the databases. The research was performed using the search strategy that included 'Dental plaque' OR 'oral care' OR 'tooth' OR 'plaque' OR 'mouth care' OR 'Chlorhexidine Gluconate (CHX)' OR 'toothbrushing' OR 'oral' AND any word = ('ventilator-associated pneumonia' OR 'ventilator acquired pneumonia') AND any word = ( 'ICU' OR 'Intensive care' OR 'ITU' OR 'ventilator' OR 'ventilation' OR 'MV' OR 'intubated' OR 'mechanical'). The search of the databases yielded successful results; however, additional references were identified through a search of Google Scholar and websites for professional organizations, including the Intensive Care Society and critical care.

\section{Background}

The 2008 guidelines of the National Institute for Health and Clinical Excellence (NICE) were used. Besides this, the National Patient Safety Agency (NPSA) guidelines were also used. This promotes the use of CHX for oral care, as part of the VAP care bundle. It highlights the administration of CHX as an ointment or in a solution, with broad-spectrum antibacterial action. The formation of bacterial colonies on the surface of the teeth is referred to as dental plaque. The plaque has a sophisticated and continuously changing conformation and expands across the surface of the teeth in the absence of mechanical disturbance (Fourrier et al., 2010). Aspiration can move these bacteria down the respiratory tract and into the lungs; this leads to pneumonia (Scannapieco, 2007; Grap et al., 2011). However, the link between oral hygiene and VAP incidence has not been fully explored (Grap, 2009), as only a few studies have reported successful limitations of VAP (Munro et al., 2009). The bacteria responsible for VAP have been identified in dental plaque (Munro et al., 2009), which suggests that the presence of plaque 
increases the VAP risk.

\section{Critical Appraisal of the Literature}

\subsection{Chlorhexidine versus Brushing Teeth}

The first research reviewed investigated VAP avoidance through the use of Chlorhexidine and teeth brushing. Munro et al. (2009) performed a study to assess whether two methods of oral care, i.e., brushing teeth and a $0.12 \%$ solution of Chlorhexidine, decline VAP risk within the initial seven intubation days for ventilated patients. These participants were ICU adults who were critically ill and had been intubated within 24 hours of admission. It recruited 547 patients and randomly assigned them to four groups. This study used a clinical randomized controlled trial, a $2 \times 2$ factorial design. Grove et al. (2013) argue that this design type is most effective for determining the value of interventions given its minimization of the potential bias. Correspondingly, following the design correct implementation, it serves as a benchmark for testing health care intervention effectiveness.

In Munro et al. (2009) study, participants were divided randomly into four groups, where one group was given a $0.12 \%$ solution Chlorhexidine oral swab two times per day $(\mathrm{n}=119)$; some had their teeth brushed three times per day $(\mathrm{n}=113)$; some had a mixture of toothbrushing and Chlorhexidine $(\mathrm{n}=116)$, and one was a control group $(\mathrm{n}=123)$. For determining ventilator-associated pneumonia, Clinical Pulmonary Infection Score (CPIS) was used. The sample size was determined statistically. Polit and Beck (2012) observed that this is a strength in research as it is considered essential in quantitative research to realize a statistical sample size. The collection of data was held from the day of admission until intubation seventh day, or when the patient was extubated (Munro et al., 2009). It must be noted that results from unexpected early extubations; the sample size was reduced between days five and seven, which makes drawing strong conclusions. Subsequently, the researchers implemented the data for up to day three, only for an evaluation of their investigation's outcomes.

These outcomes showed no major difference concerning the VAP development between the Chlorhexidine ( $\mathrm{P}=.29)$ and toothbrushing $(\mathrm{P}=.95)$ groups, in comparison to the Chlorhexidine group alone. The intervention's failure to create statistically significant effects may be the result of an insufficiently large sample size, which, as Polit and Beck (2010) note, is necessary to identify significant effects of interventions. However, the study demonstrated that twice daily application of $0.12 \%$ Chlorhexidine with an oral swab significantly decreases the VAP incidence by day three (CPIS $\geq 6$ ) for the patients that had CPIS level of $<6$ at baseline $(P=.006)$. Tooth-brushing did not affect CPIS, neither it enhances the effect of chlorhexidine (Munro et al., 2009). The study strength includes its' increased toothbrushing to evaluate alternative methods to decrease the incidence of VAP. The random selection of the sample adds additional strengths to the results (Creswell, 2009). However, the study improved as initially there were only 547 patients, and by day three, only 249 patients remained intubated. Roberts \& Moule (2011) also showed that VAP reduced as a result of chlorhexidine, where the effectiveness of brushing teeth was found to be low. Whereas, Khan et al. (2017) study, with a follow-up period of two months, reported a reduction of the ventilator-associated pneumonia risk through thrice-daily oral care using a toothbrush and $0.2 \%$ chlorhexidine gluconate. However, the small follow-up period serves as a weak point.

De Lacerda Vidal et al. (2017) study also compared the two using a prospective, randomized trial. It included 213 patients that were admitted in the critical care unit, which were distributed into two groups; 108 to control group and 105 to the intervention group. The results showed no statistically significant results for the use of $0.12 \%$ chlorhexidine gel and toothbrushing. The same results were also found for the length of stay at ICU as well as mortality rate.

\subsection{Chlorhexidine Oral Swab}

The randomized study of Özçaka et al. (2012) was considered which conducted a double-blind, controlled experiment to investigate the efficacy of a $0.2 \%$ Chlorhexidine gluconate (CHX) with an oral swab reduces the incidence of ventilator-associated pneumonia in ICU patients. Through the statistical determination of the sample size (Creswell, 2009), examined sixty-one patients who were mechanically ventilated with predicted ventilation for at least 48 hours after admission. Polit and Beck (2010) note it as a strength of this research. In this intervention group, twenty-nine patients were given a $30 \mathrm{ml}$ oral swab of $0.2 \%$ Chlorhexidine gluconate four times a day. Whereas, the control group consisted of thirty-two patients with an oral swab of normal saline. Moule and Goodman (2014) assert that study validity depends on the randomization of the intervention and control of patient groups.

Parahoo (2014) argues that one method of eradicating bias is to use a computerized randomization system. In contrast, Burns and Grove (2010) produced a rigorous list of inclusion, and exclusion criteria propose a similarity between the two groups share characteristics concerning the factor of prognosis. It used clinical periodontal 
measurements and specimens from the lower respiratory tract for further analysis after the detection of the initial VAP stages (Özçaka et al., 2012). It found that control group had significantly higher VAP $(68.8 \%)$ when compared with the chlorhexidine group $(41.4 \%) ;(p=0.03)$ with an odds ratio of 3.12 [95\% confidence interval $=1.09-8.91$ ] (Özçaka et al., 2012). The study strength is its use of randomized, double-blind, controlled study methods to divide the patients into different groups. This avoided bias, given the lack of background knowledge about the patients and their diseases. However, small size serves as its weak point, with only sixty-one patients. This affects the results' reliability because it leads to higher variability and biases (Parahoo, 2014). Therefore, additional studies with larger samples are needed to validate the findings.

A third analysis is of Sona et al. (2009), an observational post-intervention group study of 12 months conducted from 2004 to 2005. The focus of this study was on the implementation of oral care protocol. For this, the researcher studies the pre-intervention group for determining the infections number concerning the ventilator days. The study considered the protocol for oral care, comprising of chlorhexidine on VAP for the surgical intensive care unit (SICU) patients that were mechanically ventilated. This study excluded patients with CHX allergies or mouth ulcer It included mechanically ventilated patients; whose comparison was held following the implementation of the oral protocol. This was carried out at a university-associated teaching hospital named Barnes-Jewish Hospital.

The pre-intervention group included 777 mechanically ventilated patients while 871 mechanically ventilated patients were present in the post-intervention group. This sample size is determined statistically and is explicitly stated. According to Polit and Beck (2012), this serves as a study strength given its significance to realize a statistical sample size in quantitative studies. Nonetheless, as Polit and Beck (2012) note, it as a weakness as no detailed explanation of sample selection is given. Throughout the study, an infection control team followed the study patients, which tracked VAP using the National Nosocomial Infections Surveillance System (Sona et al., 2009).

The RN (Registered Nurses) and the units' physicians were trained. The training of the two clinical nurse specialists (CNSs) was held who trained the remaining staff as well as monitored the study. As per the protocol, the teeth were cleaned after every twelve hours for at least one to two minutes using a toothbrush and paste. The toothpaste comprised of sodium monoflurophosphate $0.7 \%$. Following the teeth brushing of the patients, the oral cavity was rinsed using tap water (in an irrigating syringe), following patients' mouth suction. Lastly, CHX (15mL) was applied with the help of a foam sponge. The statistical significance (p-value) was found to be 0.04 . This shows the reduction in the VAP infection rate for the pre-intervention and the post-intervention periods (Sona et al., 2009).

The second last research study was illustrated by Garcia et al. (2009) which assessed the implementation consequences of comprehensive oral and dental care systems on the VAP rate. Patients $(n=759)$ who were 18 years of age or above, with hospitalization between January 1, 2001, and December 31, 2004, and to a 10-bed medical intensive care unit (MICU) were included in case they were intubated for over 48 hours. The sampling type was convenience sampling, but the researchers do not clearly state this. Convenience sampling is the least effective non-probability sampling type (Gerrish and Lathlean, 2015). Also, a convenience sample affects the generalisability of the results as the sample may not be representative of the population as a whole due to the lack of randomization (Ary et al., 2009). Patients in the research were assessed for the incidence of VAP until (i) patients, in the MICU, were weaned off from mechanical ventilation, (ii) a period of 48 hours after their transfer, and/or (iii) their death. These were used as the regulated treatment protocols for the four years. This comprised weekly change in the ventilator circuit, daily replacement of heat moisture conversation apparatus, a closed suctioning system, 30-degree angling of the bed head, prevention of peptic ulcer disease; and a vigorous weaning procedure. No chlorhexidine was orally delivered.

From January 1, 2003, and December 31, 2004 (intervention phase), application of an inclusive oral and dental care system was done (Garcia et al., 2009). This system reduced oral cavity accumulation through secretions control following the endotracheal tube insertion, in addition to plaque reducing on teeth surface. The methodology of this procedure involved oropharyngeal suctioning in every 6 hours; tissue and gums oral cleaning every 4 hours or as required; and brushing of teeth twice a day. The findings showed that in the intervention phase, VAP incidence declined to about $33.3 \%$. Following the oral care regimen implementation, mechanical ventilation and stay length decreased.

Sharif-Abdullah et al. (2016) also assessed the effectiveness of chlorhexidine for the routine oral care concerning edentulous geriatric inpatients. It conducted a Brief Oral Health Status Examination following an oral swab, which lasted for seven days. It shows that the use of Chlorhexidine helped reduced the oral colonization in contrast to the routine oral care using thymol $(\mathrm{p}<0.001)$. Garcia et al. (2009) four-year study yielded four significant findings. It 
showed decline in VAP probability and incidence through the multidimensional procedure application that comprises a daily oral appraisal combined with methods and tools specially designed for reducing oral bacteria in the subglottic region and on the teeth while stating that infection can be prevented and maintained for substantial periods. It also found that despite the standard use of other evidence-based interventions, such as elevating the head of the bed, and prophylaxis of stress ulcers, zero occurrences of VAP was not observed before the implementation of this unique procedure. It revealed that a comprehensive program of oral-dental care reduces the time for mechanical ventilation, i.e., 7.2 days to 5.1 days. In Garcia et al.'s study, it would have been beneficial to assess the patient's outcomes randomly. In an intensive care unit, the patient's nature varied, and increasing probability for extenuating circumstances, such as emergent intubation. Overall, though, the findings of this study support the fact that oral care regiment reduces and prevents VAP.

\section{Recommendations}

One way to implement these findings is to educate staff and raise awareness of the significance of oral care and the application of $0.12 \%$ Chlorhexidine, particularly to patients on ventilators. To suitably apply the evidence-based research and recommendations, every ICU's unit educator needs to use evidence-based oral care by educating nurses to implement $0.12 \%$ Chlorhexidine for any patient on a ventilator (Cason et al., 2007). Charge nurses should also ensure that employees and departments are implementing vigorous standards of oral care by applying $0.12 \%$ Chlorhexidine to mechanically ventilated patients. If nurses and staff have a strong awareness of VAP and what leads to it, and their skills are contemporary, this will enable them to adhere to hospital procedures. Gupta et al. (2016) also recommend that interventions for the e4stablishing the significance of oral care must be established among nurses for improving patients' quality of life, resulting in reducing financial recourses.

Furthermore, nurses need to develop evidence-based approaches for the daily care of patients on ventilators. As the use of $0.12 \%$ Chlorhexidine for mechanically ventilated patients in ICUs has demonstrably decreased the incidence of VAP, healthcare professionals should start to implement contemporary methods to avoid complications (Babcock et al., 2013). Thus, it is recommended that ventilated patients should receive oral care involving $0.12 \%$ Chlorhexidine.

It can be stated that ensuring the utmost treatment quality of the patient is the responsibility of the nurse. In case no policies exist for VAP, the nurses should stand for its application using the best evidence-based approach. Also, they must continuously engage in learning activities and enrich their knowledge for improving the patient's outcomes. Besides, acritical care units should implement protocols and drastic measures for detecting ventilated patients and treating VAP.

\section{Conclusion}

This critical review depicted that the development of ventilator-associated pneumonia (VAP) significantly reduced in cases where $0.12 \%$ Chlorhexidine is applied to mechanically ventilated ICU patients. The reviewed studies presented that oral care with $0.12 \%$ Chlorhexidine can decrease the occurrence of bacterial growth. Also, implementation of good oral care has affirmative outcomes for mechanically ventilated patients, such as improving patient outcomes, decreasing the lengths of patients' hospital stays, and decreasing the cost of health care. In fact, in certain other countries, a higher concentration of Chlorhexidine is used $(0.2 \%)$, but due to the Food and Drugs Administration (FDA) regulations, only $0.12 \%$ Chlorhexidine is recommended. Future studies are needed to assess the exact ways in which $0.12 \%$ Chlorhexidine prevents VAP, using more controlled trials and larger population groups.

\section{Acknowledgments}

The author is very thankful to all the associated personnel in any reference that contributed to/for this research. Further, this research holds no conflict of interest and is not funded through any source.

\section{Competing Interests Statement}

The authors declare that there are no competing or potential conflicts of interest.

\section{References}

Asehnoune, K., Seguin, P., Allary, J., Feuillet, F., Lasocki, S., Cook, F., ... \& Perrigault, P. F. (2014). Hydrocortisone and fludrocortisone for prevention of hospital-acquired pneumonia in patients with severe traumatic brain injury (Corti-TC): a double-blind, multicentre phase 3, randomised placebo-controlled trial. The Lancet Respiratory medicine, 2(9), 706-716. https://doi.org/10.1016/S2213-2600(14)70144-4

Babcock, H. M., Zack, J. E., Garrison, T., Trovillon, E., Jones, M., Fraser, V., \& Kollef, M. (2013) An Educational Intervention to Reduce Ventilator-Associated Pneumonia in an Integrated Health System: A Comparison of 
Effects. CHEST, 125 (6). pp. 2224-2231. https://doi.org/10.1378/chest.125.6.2224

Burns, N., \& Grove, S. (2009). The practice of nursing research: Conduct, critique, and utilization (5th ed.). Philadelphia: Saunders.

Cason, C., Saunders, S. \& Broome, L. (2007) Nurses' implementation of guidelines for ventilator-associated pneumonia from the centre for disease control and prevention. American Journal of Critical Care, 16(2), 28-36. https://doi.org/10.4037/ajcc2007.16.1.28

Creswell, J. W. (2009). Research design: Qualitative, quantitative, and mixed methods approaches (3rd ed.). Thousand Oaks, CA: Sage.

De Lacerda Vidal, C. F., de Lacerda Vidal, A. K., de Moura Monteiro, J. G., Cavalcanti, A., da Costa Henriques, A. P., Oliveira, M., ... \& Gomes, B. (2017). Impact of oral hygiene involving toothbrushing versus chlorhexidine in the prevention of ventilator-associated pneumonia: a randomized study. BMC infectious diseases, 17(1), 112. https://doi.org/10.1186/s12879-017-2188-0

Fourrier, F., Cau-Pottier, E., Boutigny, H., Roussel-Delvallez, M., Jourdain, M. \& Chopin, C. (2010) Effects of dental plaque antiseptic decontamination on bacterial colonization and nosocomial infections in critically ill patients. Intensive Care Medicine, 26(2), 1239-1247. https://doi.org/10.1007/s001340000585

Garcia, R., Jendresky, L., Colbert, L., Bailey, A., Zaman, M., \& Majumder, M. (2009) Reducing ventilator-associated pneumonia through advanced oral-dental care: A 48-month study. American Journal of Critical Care, 18(6), 523-531. https://doi.org/10.4037/ajcc2009311

Gerrish, K., \& Lathlean, J. (2015). The research process in nursing (7th ed.). Chichester, West Sussex, UK: Wiley Blackwell.

Grap, M. (2009). Not-so-Trivial Pursuit: Mechanical Ventilation Risk Reduction. American Association of Critical Care Nurses, 18(4), 299-309. https://doi.org/10.4037/ajcc2009724

Grap, M., Munro, C. L., Elswick, R. K., Sessler, C. N., \& Ward, K.R. (2011) Duration of action of a single, early oral application of chlorhexidine on oral microbial flora in mechanically ventilated patients: a pilot study. Heart \& Lung: The Journal of Acute and Critical Care, 33(2). 83-91. https://doi.org/10.1016/j.hrtlng.2003.12.004

Gupta, A., Gupta, A., Singh, T. K., \& Saxsena, A. (2016). Role of oral care to prevent VAP in mechanically ventilated Intensive Care Unit patients. Saudi journal of anaesthesia, 10(1), 95. https://doi.org/10.4103/1658-354X.169484

Hua, F., Xie, H., Worthington, H. V., Furness, S., Zhang, Q., \& Li, C. (2016). Oral hygiene care for critically ill patients to prevent ventilator-associated pneumonia. Cochrane Database of Systematic Reviews, (10). https://doi.org/10.1002/14651858.CD008367.pub3

Khan, M., Mohamed, Z., Ali, S., Saddki, N., Masudi, S. A. M., \& Sukminingrum, N. (2017). Oral Care Effect on Intubated Patient with 0.2 per cent Chlorhexidine Gluconate and Tooth Brushing in Intensive Care Unit. Journal of advanced oral research, 8(1-2), 26-33. https://doi.org/10.1177/2229411217729099

Mauk, K. L. (2010). Gerontological nursing: competencies for care. Sudbury, MA. Jones and Bartlett Publishers, LLC.

McCuske, K., \& Gunadyin, S. (2015). Research using qualitative, quantitative or mixed methods and choice based on the research. Journal article perfusion, 30(7). 537-542. https://doi.org/10.1177/0267659114559116

Moule, P., \& Goodman, M. (2014). Nursing research: an introduction (2nd ed.). Los Angeles, California: SAGE.

Munro, C., Grap, M., Jones, D., McClish, D. \& Sessler, C. (2009) Chlorhexidine, Toothbrushing and Preventing Ventilator-Associated Pneumonia in Critically Ill adults. American Journal of Critical Care, 18(2). 428-437. https://doi.org/10.4037/ajcc2009792

National Institute for Health and Clinical Excellence/National Patient Safety Agency (NICE)/(NPSA). (2008). Press release NICE/NPSA issues patient safety solution guidance to reduce the risk of pneumonia in patients on a ventilator.

Özçaka, Ö., Başoğlu, Ö. K., Buduneli, N., Taşbakan, M. S., Bacakoğlu, F. \& Kinane, D. F. (2012) Chlorhexidine decreases the risk of ventilator-associated pneumonia in intensive care unit patients: A randomized clinical trial. Journal of Periodontal Research, 47(3). 584-592. https://doi.org/10.1111/j.1600-0765.2012.01470.x 
Parahoo, K. (2014). Nursing Research: Principles, Process and Issues (3rd ed.). Palgrave Macmillan Limited.

Polit, D. F., \& Beck, C. T. (2010). Nursing research: Principles and methods (7th ed.). Philadelphia: Lippincott Williams \& Wilkins.

Roberts, N., \& Moule, P. (2011). Chlorhexidine and tooth-brushing as prevention strategies in reducing ventilatorassociated pneumonia rates. Nursing in critical care, 16(6), 295-302. https://doi.org/10.1111/j.1478-5153.2011.00465.x

Rosenthal, V. D., Al-Abdely, H. M., El-Kholy, A. A., AlKhawaja, S. A. A., Leblebicioglu, H., Mehta, Y., ... \& Salgado-Yepez, E. (2016). International Nosocomial Infection Control Consortium report, data summary of 50 countries for 2010-2015: Device-associated module. American journal of infection control, 44(12), 1495-1504. https://doi.org/10.1016/j.ajic.2016.08.007

Scannapieco, F. A. (2011) Role of oral bacteria in respiratory infection. Journal of Periodontol, 70(7). 793-802. https://doi.org/10.1902/jop.1999.70.7.793

Scannapieco, F.A., Jihnee, Y., Radhavendran, K., Vacanti, A., Owens, S., Wood, K. \& Mylotte, J.M. (2009) A randomised controlled trial of chlorhexidine gluconate on oral bacterial pathogens in mechanically ventilated patients. Critical Care, 13(3). 427-431. https://doi.org/10.1186/cc7967

Sharif-Abdullah, S. S. B., Chong, M. C., Surindar-Kaur, S. S., Kamaruzzaman, S. B., \& Ng, K. H. (2016). The effect of chlorhexidine in reducing oral colonisation in geriatric patients: a randomised controlled trial. Singapore medical journal, 57(5), 262. https://doi.org/10.11622/smedj.2016091

Sona, C. S., Zack, J. E., Schallom, M. E., McSweeney, M., McMullen, K., Thomas, J., ... \& Schuerer, D. J. (2009) The impact of a simple, low-cost oral care protocol on ventilator-associated pneumonia rates in a surgical intensive care unit. Journal of Intensive Care Medicine, 24(1), 54-62. https://doi.org/10.1177/0885066608326972

Sousa, A. S., Ferrito, C., \& Paiva, J. A. (2018). Intubation-associated pneumonia: An integrative review. Intensive and Critical Care Nursing, 44, 45-52. https://doi.org/10.1016/j.iccn.2017.08.003

Torres, A., Niederman, M. S., Chastre, J., Ewig, S., Fernandez-Vandellos, P., Hanberger, H., ... \& Paiva, J. A. (2017). International ERS/ESICM/ESCMID/ALAT guidelines for the management of hospital-acquired pneumonia and ventilator-associated pneumonia: Guidelines for the management of hospital-acquired pneumonia (HAP)/ventilator-associated pneumonia (VAP) of the European Respiratory Society (ERS), European Society of Intensive Care Medicine (ESICM), European Society of Clinical Microbiology and Infectious Diseases (ESCMID) and Asociación Latinoamericana del Tórax (ALAT). European Respiratory Journal, 50(3), 1700582. https://doi.org/10.1183/13993003.00582-2017

Wei, H. P., \& Yang, K. (2019). Effects of different oral care scrubs on ventilator-associated pneumonia prevention for machinery ventilates patient: A protocol for systematic review, evidence mapping, and network meta-analysis. Medicine, 98(12), e14923. https://doi.org/10.1097/MD.0000000000014923

Wunsch, H., Linde-Zwirble, W.T., Angus D.C., Hartman, M.E., Milbrandt, E.B., Kahn J. M. (2010) The epidemiology of mechanical ventilation use in the United States. Critical Care Medicine, 38(10). 1947 -1953. https://doi.org/10.1097/CCM.0b013e3181ef4460.

\section{Copyrights}

Copyright for this article is retained by the author(s), with first publication rights granted to the journal.

This is an open-access article distributed under the terms and conditions of the Creative Commons Attribution license (http://creativecommons.org/licenses/by/4.0/). 